\title{
Closed-loop insulin delivery
}

Manual closed-loop insulin delivery could reduce the risk of nocturnal hypoglycemia in children and adolescents with type 1 diabetes mellitus, state researchers of a new study published in the Lancet.

Continuous monitoring devices detect changes in blood glucose concentration as an aid to establish glycemic control. Subcutaneous infusion of insulin can be achieved with the use of a portable electromechanical pump, which infuses insulin at preprogrammed, slow basal rates, with patient-activated boosts at mealtimes. Closed-loop systems combine continuous glucose monitoring with insulin pumps. In contrast to the delivery of insulin at preprogrammed rates, closed-loop systems enable the administration of insulin according to real-time glucose measurements, directed by a control algorithm.

In three randomized crossover studies, the investigators assessed the safety and efficacy of overnight closed-loop insulin delivery systems as a potential candidate for the generation of the first artificial pancreas system. "The present work is a stepping stone towards home testing of closed-loop systems," says lead researcher Roman Hovorka (Addenbrooke's Hospital, Cambridge, UK). "Our focus was on the development of the control algorithm and clinical testing." At inception, the researchers designed three randomized crossover

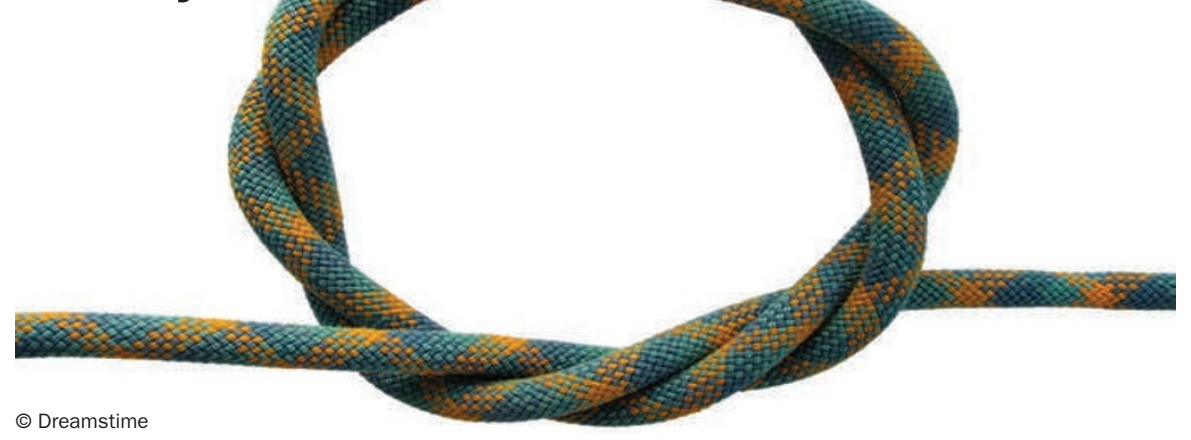

studies in 19 patients aged 5-18 years with type 1 diabetes mellitus, in which they compared closed-loop delivery with standard continuous subcutaneous insulin infusion (Artificial Pancreas Project at Cambridge [APCam] 01), closed-loop delivery after rapidly and slowly absorbed meals (APCam02) and the effects of moderate-intensity evening exercise (APCam03). Hovorka et al. decided to assess the closed-loop systems in children and adolescents, as this group of patients has only limited availability of other treatments, such as islet and pancreas transplants, yet would benefit most from improved glycemic control. Night-time glucose measurements using the closedloop system were integrated into a control algorithm every $15 \mathrm{~min}$, and the calculated insulin infusion rate was then adjusted on an insulin pump by a nurse. During control nights, the patients received insulin according to their standard insulin pump settings.
The primary outcome measure was the time for which plasma glucose concentration was $3.91-8.00 \mathrm{mmol} / \mathrm{l}$ or $\leq 3.90 \mathrm{mmol} / 1$. No differences were determined in the primary outcomes of 33 closed-loop nights versus 21 continuous infusion nights in either of the three trials. No hypoglycemic events occurred during closed-loop delivery compared with nine events during standard treatment.

Hovorka concludes, "in an incremental fashion, closed-loop systems promise to transform the treatment of type 1 diabetes mellitus." Various improvements, such as "speeding up absorption of insulin and improving accuracy and reliability of continuous monitoring devices", are, however, still needed.

\section{Linda Koch}

Original article Hovorka, R. et al. Manual closed-loop insulin delivery in children and adolescents with type 1 diabetes: a phase 2 randomised crossover trial. Lancet 375, 743-751 (2010) 